\title{
Data quality audit of the arthroplasty clinical outcomes registry NSW
}

\author{
Kurt G Seagrave ${ }^{1}$, Justine Naylor ${ }^{1,2,3}$, Elizabeth Armstrong ${ }^{1,2}$, Kwong-Ming Leong $^{1}$, Joseph Descallar ${ }^{1,4}$ \\ and lan A Harris ${ }^{1,2,3^{*}}$
}

\begin{abstract}
Background: The Arthroplasty Clinical Outcomes Registry NSW (ACORN) was initiated in 2012. ACORN is a registry piloting within NSW, Australia with several participating hospitals; it aims to monitor patient-centred outcomes and post-surgical complications after total hip and knee arthroplasty. Using retrospective audit methodology, we aimed to investigate the completeness and accuracy of data in ACORN.
\end{abstract}

Methods: We undertook a reabstracting audit of 100 clinical records of patients who underwent surgery in 2012/2013 (50 each from hospitals A and B). These records represented 27\% (100/367) of patient entries in the ACORN registry, all of which were collected at either hospital A or hospital B. Firstly, data completeness was determined by identifying the proportion of missing data in the original data pro forma. Secondly, accuracy of the initial data extraction was determined by comparing these data to reabstracted data collated by an auditor blind to the outcomes of the initial extraction. Inaccuracies were ascertained to be a disagreement between categorical variables and for continuous data, a pre-determined window of error was established. Benchmarks for data completeness and accuracy were set at 95.0\%; kappa and intraclass coefficient (ICC) calculations were also utilised to supplement this analysis. In addition, registry completeness (the percentage capture of eligible patients) was also determined as part of the data quality analysis.

Results: Completeness and accuracy of submitted datasets were evaluated to be 99.0\% (1259/1272) and 94.0\% (2159/ 2296) respectively for Hospital A, and 99.3\% (1589/1600) and 96.1\% (2444/2542) for Hospital B. The majority of accuracy discrepancies pertained to medical history data. For Hospital A, 57.1\% (28/49) of variables met the accuracy benchmark of 95\%; 74.5\% (38/51) of variables in Hospital B met this benchmark. Of the number of patients eligible for inclusion in the registry, 93.5\% (660/706) were found to be included.

Conclusion: Levels of data completeness and accuracy were found to be high in the submitted datasets for both hospitals. However, important deficits were identified in the accuracy of patient comorbidities. More specific and clear data definitions, and a more thorough examination of medical records would be possible methods to improve the accuracy of deficient areas.

Keywords: Medical audit, Medical records, Arthroplasty, Registries

\section{Background}

Outcomes data are increasingly being used to improve the quality of care delivered by health services. The Australian Orthopaedic Association National Joint Replacement Registry (AOANJRR) commenced in 1999. As a national registry, it functions to monitor a specific outcome time to

\footnotetext{
* Correspondence: iaharris1@gmail.com

'South Western Sydney Clinical School, Faculty of Medicine, UNSW Australia, Liverpool Hospital, Liverpool, NSW 2170, Australia

${ }^{2}$ Whitlam Orthopaedic Research Centre, Ingham Institute for Applied Medical Research, 1 Campbell St, Liverpool, NSW 2170, Australia

Full list of author information is available at the end of the article
}

first revision of joint arthroplasty, and the registry has been successful in this aspect $[1,2]$.

However, the AOANJRR does not provide information on the impact of arthroplasty on patient-centred outcomes (such as pain, function and quality of life), nor does it monitor specific complications post-surgery beyond prosthesis revision and mortality. In order to fill this knowledge gap, the Whitlam Orthopaedic Research Centre initiated the Arthroplasty Clinical Outcomes Registry NSW (ACORN) in 2012. This clinical registry receives information from participating hospitals, and utilises outcome 
tools such as the EQ-5D-5 L [3] and the Oxford Hip and Knee scores [4], and integrates concepts borrowed from international hip and knee arthroplasty registries $[5,6]$, other Australian outcomes registries [1], and the English patient-reported outcome measures (PROMs) movement [7]. ACORN's focus on PROMs reflects the belief that patient post-operative health status and quality of life play a significant role in decision making and outcome assessment of joint replacement surgery.

For registry data to be used in the planning, monitoring and improvement of the quality of healthcare, detailed data of sufficient quality are required [8]. Such quality may be analysed in terms of completeness and accuracy. Completeness can be assessed by the coverage of total cases on which the registry is able to collect information, or with reference to missing data present in the registry (which will be used as the definition of data completeness in this report). Accuracy is a measure of correctness of each item of data collected $[8,9]$.

Whilst ACORN presently has data quality measures in place, the accuracy and completeness of data submitted using a purpose-built pro forma has not yet been substantiated. For registry data to be used to inform clinical decision-making, levels of completeness and accuracy must be assessed. In this study, using retrospective audit methodology, we aimed to determine the data quality in ACORN. Here, we randomly selected and reabstracted $27 \%$ of the records included in the ACORN database. Data quality refers to the level of completeness of the pro forma data fields, and the accuracy with which information in the original source file (the medical record) is extracted and subsequently recorded on the pro forma.

A complete patient record within ACORN contains three PROM tools: EuroQoL-5D-5 L dimensions, EuroQoL-VAS (Visual Analogue Scale) score and total for Oxford Hip or Oxford Knee Score. Of the 311 entries contributed by Hospital A (over the period 01/07/12 - 30/06/13), five contained at least one missing PROM element, representing $1.6 \%$ of the total patient records in ACORN for Hospital A. In comparison, Hospital B had 71 out of 349 records with at least one missing PROM element over the period $01 / 09 / 12$ - 30/06/13, representing $20.3 \%$ of the total Hospital B submissions: from this, 11.5\% (76/660) of patient records in ACORN contained missing PROMs data. Thus, $88.5 \%$ of patients included in ACORN completed all three baseline PROMs assessments. This is summarised in Table 1.

\section{Methods}

\section{Data source and collection}

ACORN is a prospective, longitudinal cohort registry open to public and private hospitals in Australia that undertake planned hip and knee arthroplasty. Participants are recruited on an opt-out basis. Currently, ACORN is piloting at several hospitals in NSW, and has utilised a rolling method of hospital recruitment. The data held in the registry at the time of this audit represents two foundation hospitals contributing to ACORN. Both hospitals A and B are classified within the NSW major hospitals peer group [10]. Ethical approval for this study was granted on $13 / 03 / 13$ by the Hunter New England Human Research Ethics Committee (HNE HREC) - reference no. 12/11/21/5.02.

The data collected can be divided into four main sections:

- demographic and administrative information.

- medical (co-morbid) history.

- pre-operative health status (pain, function, quality of life).

- procedure details and recovery.

The registry data collection process for both hospitals involves the completion of standardised forms, which are forwarded to the registry. The data collection process varies slightly between the two hospitals. Once each site coordinator has submitted their data (which occurs on a routine basis), a quality and completeness check is undertaken in order to identify obvious errors or missing data points, allowing for correction prior to data entry. Following data entry, data collection forms are stored and filed for use at the six-month follow-up time point.

\section{Data reabstraction}

50 patient records were randomly selected from each hospital (A and B) in the ACORN database using a computergenerated sequence. During the time period in which the audit applied (the first six months of data submission), 179 patient records were available in the ACORN database from hospital A, and 188 available in hospital B. Sampling rates were therefore $27.9 \%(50 / 179)$ and $26.6 \%(50 / 188)$ for hospitals A and B respectively. 50 patients were chosen from each hospital under the recommendations by Altman in the selection of a minimum of 50 patients for reliability and reproducibility studies [11]. Reabstraction was limited to the demographic, administrative, medical history, procedure and acute care details, and necessarily

Table 1 PROM completion and registry completeness

\begin{tabular}{llllll}
\hline Hospital & $\begin{array}{l}\text { Records with } \\
\text { Completed PROM }\end{array}$ & Total Records & Completion Rate (\%) & $\begin{array}{l}\text { No. of Relevant Cases } \\
\text { Reported at Site }\end{array}$ & Registry Completeness (\%) \\
\hline A & 306 & 311 & 98.4 & 317 & 98.1 \\
B & 278 & 349 & 79.7 & 389 & 89.7 \\
\hline
\end{tabular}


excluded the data that was patient-reported (for example, the EQ-5D-5 L and Oxford Scores). A researcher not directly involved with the ACORN data collection, entry or analysis performed the record randomisation process. Two random sequences of numbers were generated so that 50 patient records from each hospital were used. One record from hospital A was not considered for analysis as the surgical procedure detailed was excluded from the registry.

The reabstraction process involved the auditor accessing the relevant medical records at each hospital, completing the data pro forma, and then entering the data into an electronic spreadsheet (the audit dataset). A second reviewer entered the data originally submitted by the hospital into a second spreadsheet (which will be acknowledged as the original dataset). The re-entering of the original data eliminated transcription error by the previous data entry staff member as a source of inaccuracy between the first and second auditor. By doing this, measures of data completeness and accuracy were determined for the submitted datasets to ACORN. Neither the first or second auditor accessed the data spreadsheet of the other.

The information within the original dataset was then assessed for quality in terms of data completeness. Any missing cells were scored as missing data, and these were added and scored as a proportion to give a final value for the completeness of original data collection. All variables were assessed under this data quality check.

Evaluating the accuracy of the original dataset was performed by direct comparison of the abstracted and reabstracted data. The variables included were co-morbid conditions, previous lower limb arthroplasty, the side and type of arthroplasty, and recovery details such as intra- and post-operative complications, donor blood, discharge destination and length of stay.

A discrepancy in any categorical variable $(n=44$ (hospital A), $n=46$ (hospital B)) was noted as a disagreement. Kappa values were calculated for categorical variables in order to account for random error when analysing the agreement between the two datasets. The following convention was used to interpret the Kappa values; <0, less than chance agreement; $0.01-0.2$, slight agreement; $0.21-0.4$, fair agreement; $0.41-0.6$, moderate agreement; $0.61-0.8$, substantial agreement; $0.81-0.99$, almost perfect agreement [12]. For scalar variables such as height and weight, a reasonable window of error, defined as an acceptable range of values for which variation would not significantly impact on data calculations, was allowed for determining discrepancies [13]. A range of \pm 1 year was permitted for age to allow for differences in dates between forms. Height was afforded $\pm 0.03 \mathrm{~m}$ and weight $\pm 5 \mathrm{~kg}$ in accordance with variation in patient reports of these variables. Length of stay was afforded \pm 1 day of discrepancy as a result of whether the data collector identified the day of admission as day 0 or 1 . Intraclass correlation calculations (ICC) (two-way random for absolute agreement) were also determined for continuous data variables [12,14]. ICC values range from 0 to 1 with 0 suggesting no agreement; and 1 as perfect agreement. Total discrepancy levels for the datasets were then determined, as well as the individual levels of discrepancy for each variable. A finite population correction was applied to the standard errors used to calculate 95\% confidence intervals for kappa and accuracy estimates, with sampling rates of $27.9 \%$ (50/179) for hospital A and 26.6\% (50/188) for hospital B.

To minimise the impact of transcription errors (as opposed to actual differences in data extraction), a check system was implemented during the data analysis process, where any unusual discrepancies in the two datasets were double checked against the original and audit forms simultaneously.

In addition, registry completeness data were determined to supplement the analysis of data quality. This numerator was the total number of patient records in ACORN in a defined period, with the denominator being the total number of hip and knee replacements performed at hospitals $\mathrm{A}$ and $\mathrm{B}$. The denominator was determined by reviewing the hospital administrative database to report the total number of primary and revision hip and knee replacements performed by each hospital for the equivalent period. The administrative databases are independently managed from the ACORN project.

\section{Results}

\section{Registry completeness}

It was determined that 317 hip and knee arthroplasties were performed at Hospital A for the period 01/07/12 $30 / 06 / 13$. ACORN contains data on 311 of these cases. From 01/09/12 - 30/06/13, Hospital B reported 389 hip and knee arthroplasties. ACORN holds 349 of these cases. In summary, the ACORN registry captured 98.1\% (311/317) of cases from Hospital A, and 89.7\% (349/389) of cases of Hospital B for the defined period. This represents $93.5 \%(660 / 706)$ of the total number of patients eligible for inclusion in the registry. Table 1 summarises this data.

Patient records with no missing data and no discrepancies accounted for $10.2 \%$ (5/49) of records in Hospital A, and for $8.0 \%(4 / 50)$ of records in Hospital B, as shown in Table 2 .

\section{Data completeness}

Thirteen missing data entries were identified for Hospital A. This provided a final data completeness value of $99.0 \%$ (1259/1272 total sections completed over 49 patient records). For Hospital B, data completeness was $99.3 \%$ (1589/1600 total sections completed over 50 patient records) on account of 11 missing entries. Missing data 
Table 2 Number of complete and accurate records

\begin{tabular}{lllll}
\hline Hospital & $\begin{array}{l}\text { No. of Records } \\
\text { Reabstracted }\end{array}$ & $\begin{array}{l}\text { No. of Records with Discrepancy } \\
\text { or Missing Data }\end{array}$ & $\begin{array}{l}\text { No. of Records without Discrepancy } \\
\text { or Missing Data }\end{array}$ & $\begin{array}{l}\text { Percentage of Records } \\
\text { Error-Free (\%) }\end{array}$ \\
\hline A & 49 & 44 & 5 & 10.2 \\
B & 50 & 46 & 4 & 8.0 \\
\hline
\end{tabular}

were not restricted to any variables in particular, and were spread evenly throughout the dataset.

\section{Data accuracy}

For Hospital A, 137 discrepancies were identified out of a possible 2296 matching entries $(40 \times 49+4 \times 27$ categorical and $5 \times 49$ scalar variables, minus missing data). This provided a final accuracy measurement of 94.0\% (2159/2296) for Hospital A. Across the 49 variables in Hospital A, 28 variables achieved the accuracy benchmark (95\%), representing $57.1 \%(28 / 49)$ of the total as shown in Table 3. Variables with $<85 \%$ agreement (discrepancy rates above 15\%) included those in the comorbidity field "hypertension", "previous THR/TKR" and post-operative outcomes "postoperative complications", "other" and "donor blood units". Twenty variables achieved substantial or more $(\geq 0.6)$ kappa values. Lower-range kappa values (below 0.40) were calculated for the comorbidity "other lower limb arthritis" (attributable to poor agreement), and the post-operative complications "suspected superficial wound infection" and "other" (a result of low incidence levels in the original dataset). Agreement for the variable "post-operative complications" demonstrated the lowest agreement value (73.5\% - 36/49 records agreed). 71 discrepancies in the medical history data section made up $51.8 \%$ (71/137) of total discrepancies found. "See Additional file 1 - Hospital A (1) and (2)".

In summary, $89.8 \%(44 / 49)$ of patient entries assessed for hospital A contained at least one data field discrepancy or missing data field. $94.0 \%$ (2159/2296) of individual data fields assessed were determined to be accurate. $57.1 \%$ $(28 / 49)$ of variables assessed met the $95.0 \%$ benchmark for data accuracy.

The accuracy of data collected from Hospital B was determined to be $96.1 \%(2444 / 2542)$ due to 98 discrepancies identified from a possible 2542 matching entries ( $46 \times 49$ categorical $+5 \times 50$ continuous data, minus missing data). Discrepancy rates over $15.0 \%$ were noted in the comorbidities "GIT", "hyperlipidaemia", "respiratory" and "lower back problems". Low-range kappa values were noted in the comorbidities "GIT" and "hyperlipidaemia" (on account of poor agreement); the comorbidity "CNS" and post-operative complications "bladder retention" and "other" rendered low-range kappa values on account of low incidence. Of the 51 variables tested, 38 met the $95 \%$ accuracy benchmark which represents $74.5 \%$ of the total (38/51) (as shown in Table 3). The comorbidity "hyperlipidaemia" marked the lowest agreement value across all variables (70.0\% - 35/50 records agreed). The majority of discrepancies noted were present in the medical history data section (77.6\% (76/98) of total discrepancies). "See Additional file 1 - Hospital B (1) and (2)".

92.0\% (42/50) of patient entries assessed in hospital B contained at least one data field discrepancy or missing data field. $96.1 \%(2444 / 2542)$ of individual data fields assessed were determined to be accurate. $74.5 \%(38 / 51)$ of variables assessed met the $95.0 \%$ benchmark for data accuracy.

Variables that met the accuracy benchmark for both hospitals included the comorbid condition "diabetes", the surgery type and side, and the majority of post-operative complications. Discrepancy rates over $10.0 \%$ for both hospitals were noted in the co-morbid conditions "heart", "hypertension", "hyperlipidaemia", "GIT" and "lower back problems". The majority of scalar variables for both hospitals measured an ICC greater than 0.90, with only "donor blood units" for Hospital A deviating from this trend ( $\mathrm{ICC}=0.580$ ) on account of poor agreement.

\section{Discussion}

The levels of data completeness for both hospitals were high, with forms being completely filled in the majority of cases. Missing data appeared to be random. The consistently high levels of data completeness indicate that current systems in place to address potential issues have been successful [15].

The North American Association of Central Cancer Registries (NAACRR) have determined benchmarks of $\geq 90 \%$ ( silver certification) and $\geq 95 \%$ (gold certification) to be used for determining the quality of coverage of

Table 3 Discrepancy benchmarks in hospitals A and B

\begin{tabular}{lllll}
\hline Hospital & $\begin{array}{l}\text { No. of Variables } \\
\text { Reviewed }\end{array}$ & $\begin{array}{l}\text { No. of Variables with } \\
\mathbf{2 9 5 \%} \text { Accuracy }\end{array}$ & $\begin{array}{l}\text { No. of Variables with } \\
<95 \% \text { Accuracy }\end{array}$ & $\begin{array}{l}\text { Percentage of Variables Meeting } \\
\text { Accuracy Benchmark (\%) }\end{array}$ \\
\hline A & 49 & 28 & 21 & 57.1 \\
B & 51 & 38 & 13 & 74.5 \\
\hline
\end{tabular}


a cancer registry [16]. From this, the research team aimed to achieve a final accuracy value of $95 \%$. Hospital A just falls short by $1.0 \%$, whilst Hospital B exceeds this benchmark by $1.1 \%$ [17].

There are several factors most likely to have impacted on the discrepancy levels for both hospitals. It is important to note that as part of the data analysis process of determining discrepancies, it was assumed that the auditors' account was correct when a mismatch occurred during comparison with the original dataset. However, the impact of false positives and negatives must be considered for both the auditor and original data collector, and also the limits of the retrospective study. Our results also suggest a certain difficulty in analysing some variables, which may be derived from a variety of sources, such as the patient or a clinician. These variables included patient weight and height, and their comorbid conditions. Variability in these fields was noted within the same record, which in turn required the auditor to determine which version was correct. Disorganised form arrangement in some medical records may also have contributed to the increased likelihood of discrepancies occurring during the data extrapolation process, for both the original data collection and the audited data. Hospital B was identified as having the more structured and organised medical record, allowing data extraction to be relatively straightforward in comparison to Hospital A.

This was acknowledged as one of the key factors influencing the number of variables meeting the 95\% accuracy benchmark (57.1\% of variables in Hospital A as compared to $74.5 \%$ in Hospital B).

Another issue with discrepancy levels were the definitions of the data: the registry took the opinion that comorbidities must be ongoing and of sufficient severity to be treated in some way. There is a grey-area when it comes to this definition, as conditions such as asthma and foot gout were commonly detailed in medical records but were often not included in the original data collection forms. This highlights the importance of having strict and unambiguous definitions of data to be collected, and continuously reviewing such definitions in line with evaluation of the submitted data. The higher discrepancy values for particular comorbid conditions indicate that data collection staff may require additional training or information regarding the comorbidity classifications.

For scalar variables, we left some flexibility for error specifically for height and weight values. This was due to the variability of their documentation within the same record. We allowed for what we believed to be a reasonable window for both of these; the ICC values indicate that the two datasets had an otherwise high level of agreement between them. Length of stay was allowed an error of one day on account of differences as to whether the date of surgery commenced on day 0 or 1 . The registry defines the date of surgery as day 0 , however, this definition may have been interpreted differently by clinicians responsible for the collection of data. Amendment of the way length of stay is collected, from number of days to specific dates for surgery and discharge, would allow consistent application and calculation of this data field.

As the data collection form has undergone revision, based on feedback over the implementation period of ACORN, it is expected that data collected from the earlier versions would be of less quality than that of the newer versions. Regular revisions have been implemented to address issues such as the inputting of data onto the pro forma, as well as the information to collect and how data is requested. For example, version 1 of the data collection sheet asks if the patient had ever undergone a previous TKR or THR, and also to specify the type of operation. The more current versions further require both the side (left, right or both) of operation, as well as the specific procedure undertaken. This may have contributed to the lower levels of accuracy measured in Hospital A compared to Hospital B. With implementation of more specific and clear procedures for data collection and submission, it is anticipated that newer sites contributing to ACORN will consistently submit more accurate and complete data.

The minimum data are reviewed on a defined basis to look at which data items are not collected well, need better definition or require further training in interpretation. From this audit, it is clear that the methodology in collecting comorbid data requires review, as the majority of accuracy discrepancies were focussed in this area. Data source verification is an ongoing challenge for registries and for sites. For now, we suggest that comorbid data be obtained via the patient denoting the medication they are currently taking and its purpose. This site coordinator may then verify the patient-reported data with the specialist, general practitioner and anaesthetic reviews embedded in the medical record.

In the long term, the registry aims to extract from administrative systems and involve direct database entry with validation rules included, which should result in decreased duplication of data collection and reduced error. For this to be successful, hospital data collection processes and organisation of medical records would also require improvement.

\section{Conclusions}

The results indicate that data collected in the first six months of operation of ACORN from two major hospitals in NSW have high levels of data completeness and accuracy. However, though relatively infrequent, important discrepancies occurred in important areas such as comorbid conditions and complications. For these data to usefully inform practice, improvements in collection of comorbid and complication data need to occur. Clinician 
feedback from contributing sites should be incorporated into the quality framework for the registry to improve systems and clarify definitions in order to increase levels of data completeness and accuracy in ACORN.

\section{Additional file}

\section{Additional file 1: ACORN - Variables for hospitals A and B.}

Description: contains data on accuracy, kappa and incidence values of assessed variables.

\section{Abbreviations}

ACORN: Arthroplasty Clinical Outcomes Registry NSW; AOANJRR: Australian Orthopaedic Association National Joint Replacement Registry; THR: Total hip replacement; TKR: Total knee replacement; CNS: Central nervous system; PNS: Peripheral nervous system; GIT: Gastrointestinal; NAACCR: North American Association of Central Cancer Registries; ICC: Intraclass correlation coefficient; Cl: Confidence interval; CM: Comorbidity; PC: Post-operative complication; CVS: Cardiovascular system; PE: Pulmonary embolism; DVT: Deep vein thrombosis.

\section{Competing interests}

All authors declare that they have no competing interests.

\section{Authors' contributions}

$\mathrm{H}_{1}, \mathrm{JN}$ and EA and conceived the idea of this study. KS obtained the study data under the guidance of $\mathrm{H}, J \mathrm{~N}$ and $\mathrm{EA}$. KL assisted with the data compilation and entry process. JD assisted with data analysis. All authors contributed to the interpretation of data and writing of this manuscript.

\section{Acknowledgements}

To the assistance of the ACORN Steering Committee for support over the period of this research, and Deanne Jenkin for performing the randomised selection process.

\section{Author details}

'South Western Sydney Clinical School, Faculty of Medicine, UNSW Australia, Liverpool Hospital, Liverpool, NSW 2170, Australia. ${ }^{2}$ Whitlam Orthopaedic Research Centre, Ingham Institute for Applied Medical Research, 1 Campbell St, Liverpool, NSW 2170, Australia. ${ }^{3}$ South Western Sydney Local Health District, Elizabeth St, Liverpool, NSW, Australia. ${ }^{4}$ Ingham Institute for Applied Medical Research, 1 Campbell St, Liverpool, NSW 2170, Australia.

Received: 22 June 2014 Accepted: 10 October 2014

Published online: 20 November 2014

\section{References}

1. Graves SE, Davidson D, Ingerson L, Ryan P, Griffith EC, McDermott BFJ, McElroy HJ, Pratt NL: The Australian Orthopaedic Association National Joint Replacement Registry. Med J Aust 2004, 180(5 Suppl):S31-S34.

2. Choong PFM: The challenges of joint replacement surgery in the $21 \mathrm{st}$ century. ANZ J Surg 2009, 79(10):669-671.

3. Brooks R: EuroQol: the current state of play. Health Policy 1996, 37(1):53-72.

4. Murray DW, Fitzpatrick R, Rogers K, Pandit H, Beard DJ, Carr AJ, Dawson J: The use of the Oxford hip and knee scores. J Bone Joint Surg 2007, 89-B(8):1010-1014.

5. Robertsson O, Knutson K, Lewold S, Lidgren L: The Swedish Knee Arthroplasty Register 1975-1997: An update with special emphasis on 41,223 knees operated on in 1988-1997. Acta Orthopaedica Scandinavia 2001, 72(5):503-513.

6. Kärrholm J: The Swedish Hip Arthroplasty Register ( www.shpr.se ). Acta Orthop 2010, 81(1):3-4

7. The Health and Social Care Information Centre: Provisional Monthly Patient Reported Outcome Measures (PROMs) in England - A guide to PROMs methodology. 2013:0-36.

8. Evans S, Bohensky M, Cameron P, McNeil J: A survey of Australian clinical registries: can quality of care be measured? Intern Med J 2011, 41(1a):42-48.

9. Kolling C, Simmen BR, Labek G, Goldhahn J: Key factors for a successful National Arthroplasty Register. J Bone Joint Surg 2007, 89(8):1567-1573.
10. Bureau of Health Information: Local Hospital Performance [http://www.bhi. nsw.gov.au/publications/local_hospital_performance]

11. Altman DG: Practical Statistics for Medical Research. London: Chapman \& Hall; 1991.

12. Viera AJ, Garrett JM: Understanding interobserver agreement: the kappa statistic. Fam Med 2005, 37(5):360-363.

13. Herbert MA, Prince SL, Williams JL, Magee MJ, Mack MJ: Are unaudited records from an outcomes registry database accurate? Ann Thorac Surg 2004, 77(6):1960-1964

14. Rosner B: Fundamentals of Biostatistics. Belmont, CA: Duxbury Press; 2005.

15. Barrie JL, Marsh DR: Quality of data in the Manchester orthopaedic database. Br Med J 1992, 304:159-162.

16. Thoburn KK, German RR, Lewis M, Nichols P, Ahmed F, Jackson-Thompson J: Case completeness and data accuracy in the Centers for Disease Control and Prevention's National Program of Cancer Registries. Cancer 2007, 109(8):1607-1616.

17. Laustsen J, Jensen LP, Hansen AK, Danish National Vascular R: Accuracy of clinical data in a population based vascular registry. Eur J Vasc Endovasc Surg 2004, 27(2):216-219.

doi:10.1186/s12913-014-0512-6

Cite this article as: Seagrave et al:: Data quality audit of the arthroplasty clinical outcomes registry NSW. BMC Health Services Research 2014 14:512.

\section{Submit your next manuscript to BioMed Central and take full advantage of:}

- Convenient online submission

- Thorough peer review

- No space constraints or color figure charges

- Immediate publication on acceptance

- Inclusion in PubMed, CAS, Scopus and Google Scholar

- Research which is freely available for redistribution 